Europe's Journal of Psychology 2/2009, pp. 1-2

www.ejop.org

\title{
Research that makes a difference
}

\section{By Cary L. Cooper, CBE}

Lancaster University

Given the dramatic economic times we are all living in today, it is important, more than ever before, that the research we undertake in the field of psychology is of relevance to society. The economic recession is one of the worst in recent history, and will have many consequences for the cultures that we live and work in. It will not only affect the field of occupational psychology but also clinical, developmental and neuro-cognitive psychology as well. The impact of this downturn is having consequences for individual's health and well-being, family relationships, the roles of men and women at work and in the family, and for old age as well as work opportunities begin to dry up for older workers. It is incumbent of research psychologists that our work is relevant and applied to the contemporary problems we currently face. We will always engage of blue-sky or basic psychological research, but many of the problems people face in Europe during these difficult times require apposite and effective solutions. The time has come for applied psychologists to work on the issues that really matter in understanding human behaviour in times of distress. This is a golden opportunity for us, as many of the changes that are taking place across Europe provide us with a natural laboratory for our science, and the practice of applied psychology.

There are likely to be cutbacks in a variety of the public services, whether in education or health or social services, so the focus of our work should be to identify what is happening, what we can do about it and how we can pinpoint and synchronize our interventions (and, of course, assess their impact). By being instrumental in our focus on current issues, it is likely to make a difference in our respective countries, and show the usefulness of the science of psychology. We should take this opportunity of the changing economic order to explore new areas of research for psychology, as the old adage goes "if you always do what you always did-you'll always get what you always got". I would like to encourage the 
new generation of psychological researchers and practitioners to explore the dynamics of our times, of the current turmoil that many are experiencing and develop new paradigms for research and practice. This is our challenge, or as the old Chinese proverb goes, about the importance of understanding human behaviour, "if you are planning for one year, plant rice. If you are planning for ten years, plant trees. If you are planning for a hundred years, plant people".

I hope you will enjoy this issue of the journal and look forward to seeing very timely and innovative articles in the future.

About the author:

Cary Cooper is a Director and founder of Robertson Cooper Ltd, Professor of Organisational Psychology and Health and Pro Vice Chancellor at Lancaster University. Recently awarded the Lifetime Practitioner Award from the British Psychology Society in recognition of his services to the profession, he is recognised as a world-leading expert on stress and is the media's first choice for comment on workplace issues. He is a Fellow of the British Psychological Society, The Royal Society of Arts, The Royal Society of Medicine, The Royal Society of Health and an Honorary Fellow of the Royal College of Physicians. He is also the President of the British Association for Counselling and Psychotherapy, Editor-in-Chief of the Blackwell Encyclopedia of Management and the author /editor of over 100 books. 